\title{
Regional Glacier Mapping Using Optical Satellite Data Time Series
}

\author{
Solveig Havstad Winsvold, Andreas Kääb, Member, IEEE, and Christopher Nuth
}

\begin{abstract}
The first of two Sentinel-2 satellites, launched mid2015, has similar characteristics as the Landsat TM/ETM + /OLI satellites. Together, these satellites will produce a tremendous quantity of optical images worldwide for glacier mapping, with increasing temporal coverage toward the more glacierized higher latitudes due to convergence of near-polar orbits. To exploit the potential of such near-future dense time series, methods for mapping glaciers from space should be revisited. Currently, snow and ice are typically classified from an optical satellite image using a multispectral band ratio. For each scene, mapping conditions will vary (e.g., snow, ice, and clouds) and not be equally optimal over the entire scene. The increasing amount of images makes it difficult to manually select the best glacier mapping scene as is the current practice. This work is based on the above robust image ratio method for exploiting the dense temporal image coverage. Four application scenarios using time series of Landsat type data for glacier mapping are presented. First, we synthesize an optimal band ratio image from a stack of images within one season to compensate for regional differences. The second application scenario introduces robust methods to improve automatic glacier mapping by exploiting the seasonal variation in spectral properties of snow. Third, we explore the spatio-temporal variation of glacier surface types. Finally, we show how the synthesized band ratio images from the first application scenario can be used for automatic glacier change detection. In summary, we explore automatic algorithms for glacier mapping applications that exploit the temporal signatures in the satellite data time series.
\end{abstract}

Index Terms-Glacier, Landsat, optical imaging, pixel-based mapping, time series analysis.

\section{INTRODUCTION}

G LACIER change is widely acknowledged as an indicator for changing climate [1], and numerous studies have documented rapid glacier changes in mountain regions throughout the world (e.g., [2], [3]). Remote sensing techniques are ideal for measuring glaciers on a large scale, as they cover remote glacierized areas with relatively little effort. The archive of Landsat satellite scenes is important for mapping of glaciers, especially as it represents the longest running and continuous satellite record of sufficient resolution to track glacier changes.

Manuscript received October 30, 2015; revised January 13, 2016; accepted January 25,2016 . This work was supported in part by the NCoE SVALI project (Stability and Variation of Arctic Land Ice), in part by the European Research Council under the European Union's Seventh Framework Programme (FP/2007-2013)/ERC Grant 320816, and in part by the ESA project Glaciers_cci (4000109873/14/I-NB).

The authors are with the Department of Geosciences, University of Oslo, Oslo 0316, Norway (e-mail: s.h.winsvold@geo.uio.no; a.m.kaab@geo.uio.no; christopher.nuth@geo.uio.no).

Color versions of one or more of the figures in this paper are available online at http://ieeexplore.ieee.org.

Digital Object Identifier 10.1109/JSTARS.2016.2527063
The length of the archive is now comparable to a reliable climatological baseline period of $\sim 30$ years (e.g., [4], [5]).

Medium-to-high-resolution optical satellite imagery (e.g., Landsat 4/5 TM, $7 \mathrm{ETM}+, 8 \mathrm{OLI}$, and ASTER) is frequently used for glacier mapping. Especially, glacier outlines and surface types derived from remote sensing sources are widely used in glaciological and hydrological modeling, glacier change analysis, and volume estimations (e.g., [6]-[8]). In order to account for glacier changes, glacier inventories should be retrieved in intervals of a few decades [9]. However, recent down-wasting of many glaciers in the world and the persistence of glacier change due to rising air temperatures mean that glacier inventories often require shorter mapping intervals, maybe every 5-10 years [10]. Thus, more efficient methods for automatic glacier mapping are required.

Currently the most common method for mapping glacier outlines is well established [11]-[13] using the multispectral band ratio between Red and SWIR bands (short-wave infrared band). This approach, however, relies on optimal satellite scenes with minimal snow and cloud cover. With the available optical imagery, the time period between such optimal scenes can be several years, especially in cloudy maritime areas (e.g., [14]). Other indexes and methods also rely on one optimal mapping scene, such as the band ratio between near-infrared and SWIR (e.g., [15]), the normalized difference snow index (NDSI) (e.g., [16]-[18]), and more recently, object-based image analyses (OBIA) (e.g., [19], [20]).

The first of two Sentinel-2 satellites, launched on June 23, 2015, has similar characteristics as the Landsat TM/ETM + /OLI satellites [21]-[24] (Table I, see e.g., [25] for spectral band comparison between the satellite sensors). With two Sentinel satellites in orbit, a repeat coverage of 5 days will be available at the equator. Due to the polar orbit of these satellites, the repeat coverage increases toward higher latitudes, especially when combined with Landsat 8 . The spatial resolution of 10-30 m for the relevant bands is acceptable for glacier surface classification, in particular at regional scales [26]. These medium-to-high-resolution satellite images are freely available and will promote multitemporal and multisensor-based analyses.

Snow and ice have clear spectral signatures in the optical domain [27]-[29]. Throughout the season, glaciers display a unique sequence of optical properties in a yearly repetitive pattern. The seasonal evolution of a glacier can be tracked through time as the seasonal snow melts away revealing glacier ice in the ablation area and firn in the accumulation area. In this way, higher resolution time series of satellite images will increase the range of information available for glacier 
TABLE I

DESCRIPTION OF THE LANDSAT SATELLITES AND SENTINEL-2

\begin{tabular}{lccc}
\hline \hline \multicolumn{1}{c}{ Optical satellite mission } & Landsat 4,5,7 TM/ETM+ & Landsat 8 OLI/TIRS & Sentinel 2 MSI \\
\hline Launch & 1982 (TM4), 1984 (TM5), 1999 (ETM+7) & 2013 & 2015 \\
Operational & Yes (ETM+7 SLC-off) & Yes & Yes \\
Swath width $(\mathrm{km})$ & 185 & 185 & 290 \\
Sensor type & Scanner & Pushbroom & Pushbroom \\
Revisit time & 16 -days & 16-days & 5-days (S2A and S2B) \\
Radiometric resolution & 8 -bit & 12-bit & 12 -bit \\
Spatial resolution for glacier mapping & $30 \mathrm{~m}$ & 11 & 10 and 20 m \\
No. of bands & TM4=7, TM5=7, ETM+7=8 & 12 \\
Download file size & Ca. 200-250 MB (comp.) & Ca. 1 GB (comp.) & Ca. 6 GB (comp.) \\
\hline \hline
\end{tabular}

Note: With two Sentinel-2 MSI satellites in orbit (S2A and S2B), the revisit time is 5-days around equator and better toward higher latitudes. Abbreviations: TM, thematic mapper; ETM+, enhanced thematic mapper; OLI, operational land imager; TIRS, thermal infrared sensor; MSI, multispectral instrument; SLC, scan line corrector; comp., compressed.

surfaces. In addition, each pixel on a glacier has a specific temporal signature that is often different from the surrounding off-glacier terrain, thereby creating potential for glacier classification and outline detection. Many studies have utilized multitemporal analysis, especially for glacier change detection (e.g., [4], [7], [30]). However, few studies have utilized the potential of analyzing the temporal signature on- and offglacier, and to study the dynamic response of glaciers implicitly [31]-[33]. Additionally, Landsat data stacks can be used to map persistent ice and snow cover [34].

A sequence of satellite images in time results in a threedimensional (3-D) image stack $(x, y, t)$ in which pixel values at position $(x, y)$ vary with time $(t)$. In this study, we outline two principal strategies distinguished by two time scales for exploiting glacier satellite image stacks, taking advantage of the Landsat TM/ETM+ archive.

1) The multiannual approach: Using scenes from the entire Landsat archive for selected locations, i.e., stretching over many years.

2) The seasonal approach: Time-series images from one season. We simulate higher future temporal resolution by using scenes of the same season from several adjacent years with little overall glacier change.

These time series may then be interpreted using two different methodologies.

1) Stack statistics for each pixel: The result is a composite of several surface states throughout a time period without preserving their temporal sequence. (e.g., mean, median, min., max., st. dev, etc.)

2) Chronological interpretation of the data: The evolution of the ground response is captured at each pixel sequentially ordered in time.

This study explores the potential of dense satellite image time series for glacier mapping, and the possibility to develop methods that are not dependent on subjective threshold values. Note that our aim is not to develop concrete validated processing routines but rather to exemplify and evaluate a rather un-exploited potential. The results from this type of methodology can be further explored using, e.g., PCA analysis [35], ISO-cluster unsupervised classification [36], and the Otsu's method exploiting the bi-modal distribution of the pixels in a histogram [37]. Glacier mapping in this study is defined as classifying and delineating glacier outlines and surface types. Debris-covered glaciers or sections are, however, not considered because their mapping relies on different techniques and other remote sensing sources, DEMs, and manual interpretation (e.g., [38]-[40]). In the following, we explore four application scenarios: [A] synthesizing an optimal band ratio image; [B] glacier mapping based on seasonal variations; [C] glacier surface types; and [D] glacier change analysis (capital letters [A]-[D] are used throughout the text to refer to these four scenarios). The application scenarios are discussed within each section, with an overall discussion at the end of this paper.

\section{Study Area And Datasets}

Throughout this study, we use Landsat TM/ETM+ images from three heterogeneous glacier regions in the world to cover a range of ground conditions and mapping challenges. In view of rapid ground changes related to snow fall and melt, and due to the frequent cloud cover over glacier areas, a considerable limitation of the Landsat missions is the relatively low temporal resolution (16-day revisit time). However, one can simulate higher temporal resolution using images from adjacent years (e.g., \pm 2 years) as glacier changes over such a short-time span are typically small. In this study, we test the time-series mapping potential in three glaciated regions: 1) north in the Pamirs, on the border between Tajikistan/Kyrgyzstan; 2) the Chugach Mountains in Alaska; and 3) northern Patagonia in Chile. The study sites have been selected because they cover not only cold and dry continental high mountain areas (the Pamirs), warm and wet maritime areas (Chugach Mountains), and maritime areas in the rain shadow of mountains (parts of the northern Patagonian ice field, Chile) but also pure maritime conditions (south of the northern Patagonian ice field). The focus in this study will particularly be on the Chilean and Pamir study areas as they are two opposite endmembers of typical glacier conditions in terms of climate conditions. The local Pamir study area covers a region of $344 \mathrm{~km}^{2}$, with glaciers at an altitude within approximately $2800-5500 \mathrm{~m}$ a.s.l. (centerpoint: Lat: 39.516, Lon: 70.648). The regional study area in northern Patagonia, Chile covers a region of $9450 \mathrm{~km}^{2}$ and have glaciers in the altitude range between ca. 200 and 2400 m a.s.l. (centerpoint: Lat: -47.278, Lon: -72.724$)$.

Acquisitions from two satellites together, Landsat $5 \mathrm{TM}$ and Landsat $7 \mathrm{ETM}+$, provided a denser time series over glacerized areas where the image swaths from neighboring orbits overlapped. Some images from Landsat $5 \mathrm{TM}$ are also used 
TABLE II

List of Images Used for the Seasonal Time Series (Chugach Mountains, Alaska) and a Multiannual Time Series (Northern Patagonia, Chile), Used in Application Scenario [A], [C], AND [D]

\begin{tabular}{clcccc}
\hline \hline Glacier region & Landsat ID & Sensor & Date & Year & DOY \\
\hline Chugach Mountains (Alaska) & LE70670172002245EDC00 & ETM+7 & September 2 & 2002 & 245 \\
& LE70670172002229EDC00 & ETM+7 & August 17 & 2002 & 229 \\
& LE70670172002213EDC00 & ETM+7 & August 1 & 2002 & 213 \\
& LE70670172002197EDC00 & ETM+7 & July 16 & 2002 & 197 \\
& LE70670172002165EDC00 & ETM+7 & June 14 & 2002 & 165 \\
& LE70670172002117EDC00 & ETM+7 & April 27 & 2002 & 117 \\
& LT50670172004243PAC00 & TM5 & August 31 & 2004 & 243 \\
& LT50670172004227PAC00 & TM5 & August 15 & 2004 & 227 \\
& LT50670172004195PAC00 & TM5 & July 14 & 2004 & 195 \\
\hline Northern Patagonia (Chile) & LT52310931984361AAA02 & TM5 & December 26 & 1984 & 361 \\
& LE72310932000013COA00 & ETM+7 & January 13 & 2000 & 013 \\
& LE72310932002018EDC00 & ETM+7 & January 18 & 2002 & 018 \\
& LT52310932005050COA00 & TM5 & February 19 & 2005 & 050 \\
& LT52310932011051COA00 & TM5 & February 20 & 2011 & 051 \\
\hline \hline
\end{tabular}

Note that we use only data from one Landsat path.

TABLE III

Table Summarizes the Datasets Used for Seasonal Studies Where Several Years are Simulating One Season (UsEd In APPLiCATION SCENARIOS [B] AND [C])

\begin{tabular}{|c|c|c|c|c|c|c|c|c|c|}
\hline $\begin{array}{c}\text { Glacier } \\
\text { region }\end{array}$ & Path/row & \# ETM+ 7 & Start date & End date & \# TM5 & Start date & End date & $\begin{array}{c}\text { Cloud cover } \\
\text { used }\end{array}$ & Acquisition gaps \\
\hline \multirow[t]{2}{*}{$\begin{array}{l}\text { The } \\
\text { Pamirs }\end{array}$} & $152 / 033$ & 35 & $19 / 01 / 1999$ & $11 / 05 / 2003$ & 18 & $16 / 01 / 1999$ & $16 / 09 / 2000$ & Less than $70 \%$ & $\begin{array}{l}\text { TM5 from year } \\
2001,2002,2003\end{array}$ \\
\hline & $153 / 033$ & 38 & $12 / 07 / 1999$ & $20 / 05 / 2003$ & 13 & 09/01/1999 & $11 / 10 / 2000$ & Less than $70 \%$ & $\begin{array}{l}\text { TM5 from year } \\
2001,2002,2003\end{array}$ \\
\hline \multirow[t]{2}{*}{$\begin{array}{l}\text { Northern } \\
\text { Patagonia }\end{array}$} & $231 / 093$ & 51 & $05 / 07 / 1999$ & $29 / 05 / 2003$ & 37 & $18 / 01 / 1999$ & $06 / 08 / 2002$ & All & $\begin{array}{c}\text { TM5 from year 2000, } \\
2003\end{array}$ \\
\hline & $232 / 093$ & 59 & $12 / 07 / 1999$ & $20 / 05 / 2003$ & 42 & 09/01/1999 & $13 / 08 / 2002$ & All & $\begin{array}{c}\text { TM5 from year } \\
\text { 2000, } 2003\end{array}$ \\
\hline
\end{tabular}

The total number of satellite scenes for Pamir is 104 and for Chile 189.

Note that we used data from adjacent Landsat paths. (Date format: DD/MM/YYYY). The Landsat 7 SLC failure occurred on May 31 , 2003.

before and after the investigated time period. The time series for the seasonal studies (application scenarios $[\mathrm{A}],[\mathrm{B}]$, and $[\mathrm{C}]$ ), use additional adjacent years (1999-2003) of Landsat images before failure of the scan line corrector (SLC) on Landsat 7 $\mathrm{ETM}+$ (Tables II and III) to simulate potential future seasonal resolution. The SRTM DEM data were used for defining glacier centerlines and extracting surface elevation on the glaciers in the Pamirs and Chilean datasets. Due to SRTM voids in the Chilean dataset, only those larger glaciers with little voids were used.

All imagery included in this work is Landsat level-1 terrain (L1T) corrected images (radiometrically corrected and orthorectified). However, the geometric accuracy relies on accurate ground control points and the DEM [41], and we assume that the L1T products have a precise coregistration for our use. Further, subpixel misregistration of a few scenes will not significantly affect our results due to the large number of images included in the time series. All image stacks were projected to common UTM zones and WGS84 (Pamir: UTM zone 42N, Chile: UTM zone 18S, and Alaska: UTM zone 6N). No atmospheric correction was applied as atmospheric effects have little influence on relative radiance differences between the major ground classes considered (snow and ice against bare ground, vegetation, etc.). Band ratios (or normalized differences) partly eliminate atmospheric and topographic influences when the utilized bands are affected similarly [36], [42].
The Randolph glacier inventory (RGI v. 5.0, [43]) provides glacier outlines for two of our study regions (Alaska and Chile). We use the RGI glacier outlines as background comparison for our study despite uncertainties in mapping quality and the various time stamps compared to the data in this study. Due to poor RGI quality in the Pamirs, we have derived glacier outlines using the common semiautomatic method based on the band ratio Red/SWIR and supplemented by manual digitization of debris cover using high-resolution images available from Google Earth TM as recommended by Paul et al. [44].

\section{Methodology}

All four application scenarios presented in this study build upon the particularly robust results [45] from ratio images of Landsat 5 TM and 7 ETM+

$$
R_{\text {red/SWIR }}=D N_{\text {red }} / D N_{S W I R}
$$

where DN is the digital number of the pixel in a Red or SWIR band, respectively, and $\mathrm{R}$ the ratio between both. Note, we do not include the blue band which is often included with a threshold to improve mapping in shadowed areas [46], [47]. For this band ratio method, the outline classification of clean ice and snow has an uncertainty of \pm 1 pixel (30 m) [46], [48], with larger uncertainties in shadowed areas [39]. The majority 


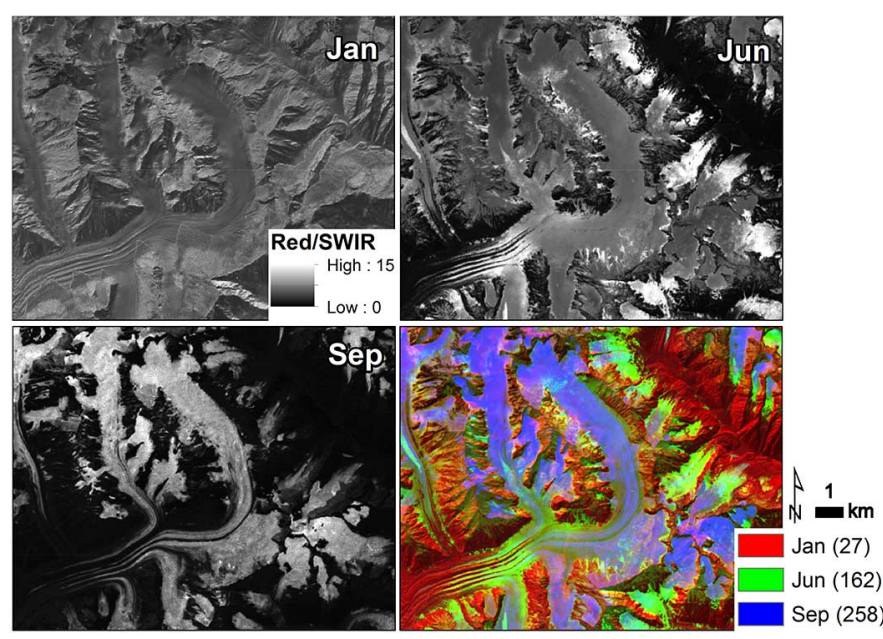

Fig. 1. False color composite of three ratio images from January, June, and September from a subset in Pamir, Tajikistan/Kyrgyzstan. The temporal signature on the glacier changes throughout the season. The colors reflect a visual illustration of changing band ratio values through time; even though a pixel might be covered by snow and ice at all time steps, it changes properties throughout the season and thus gives a variable signal. The red color reflects areas with snow only present in the January image. The green color shows snow and ice in the June image, because the spectral signal of snow and ice is higher at this time compared to the other images. The blue and purple colors are a blend of all three time steps, but September has the strongest signal due to the highest ratio values in the accumulation area. Note: The numbers in brackets are day of year.

of snow and ice pixels in a summer ratio image have values $>=2$, and the range of threshold values used in other studies is between 1.6 and 2.8 (e.g., [7], [14], [15], [40], [44]). The threshold values vary a lot in terms of study region, latitude, season, and the spectral and atmospheric conditions in the optical satellite image [12]. Furthermore, the result of this method is affected by the interpreter's subjective choice of single satellite scenes and threshold values.

For initial visual inspection of the temporal signature of glaciers, we use false color composites, in which three intraseasonal ratio images are represented by a single image, using the three RGB components, as illustrated in Fig. 1. Usually, summer glacier pixels have higher band ratio values compared to winter pixels, but the main importance is the difference in band ratio values on- and off-glacier in summer when the seasonal snow has melted away. In the following, we present methods and concepts to interpret pixel-based time series at different time scales.

A dense satellite image time series provides the opportunity to recover a large statistical sample of band ratio values, pixel by pixel, and at different time scales, either multiannually or seasonally. Here, we divide the method applied on the image time series into stack statistics and chronological order.

\section{A. Stack Statistics}

Statistics are derived from a stack of multiple rasters for each individual pixel including the mean, median, minimum, maximum, and standard deviation. Statistics are calculated using the ArcGIS software package by Esri. We use these calculations on datasets within a season to make a composite of the on- and off-glacier pixels in that season, and on a multiannual time scale, which can be used for glacier change detection analysis or for simulating higher temporal resolution by merging adjacent years together. Clouds can also be more frequent in some glacier mountain areas compared to others. A cloud mask algorithm called Fmask [49] was applied on nine of the Landsat satellite images in application scenario [A]. The Fmask algorithm performs well on snow/ice with high reflectance both for thin and thick cloud cover.

\section{B. Chronological}

Compared to glaciological mapping studies, the field of seasonal vegetation studies has a much stronger record of using dense time series to extract seasonal parameters, e.g., [50], mostly due to the applicability of lower spatial resolution satellites (e.g., MODIS and AVHRR). However, it is not until recently that time series of medium-to-high-resolution images have been exploited for land-cover mapping using curve fitting. For example, using sine curves for detection of land-cover types from normalized difference vegetation index (NDVI) [51], continuous change detection [52], and generating synthetic Landsat images [53]. Satellite-derived time series within a season are often affected by a high degree of noise in the data, for instance due to varying atmospheric conditions and clouds. Using curve fitting reduces the uncertainties and leads to more robust measures, e.g., [50]. Here, the time-series image stack is analyzed in 3-D arrays. Each image provides a band ratio snapshot at a certain time. By extracting band ratio values at a pixel ( $x$, $y)$ through time $(\mathrm{t} 1, \mathrm{t} 2, \ldots, \mathrm{tN})$, a sequential history is acquired [day of year (DOY)]. A threshold value is not manually selected for filtering each satellite image, but rather ratio values $<2$ are neglected from the analysis as cloud cover tends to result in low ratio values. As an example, we choose to fit an empirical model, a sinusoidal curve, to the band ratio time series to highlight seasonality. For comparison, a smoothed spline curve of band ratio time series, resembled a sine curve [Fig. 2(a)]. We use the trigonometric identity to fit a least-squares linear regression model to directly obtain the parameters of the sinusoidal function with a fixed angular frequency of one year ( $\omega=2 \pi / 366$ ) [Fig. 2(b)]. Thus for each pixel, we fit

$$
R(t)=a \sin (\omega t+b)=k_{1} \cos (\omega t)+k_{2} \sin (\omega t)
$$

to obtain the two coefficients $k_{1}$ and $k_{2}$. We then calculate

$$
\begin{aligned}
& a=\sqrt{\left(k_{1}^{2}+k_{2}^{2}\right)} \\
& b=\arctan 2\left(k_{1}, k_{2}\right)
\end{aligned}
$$

where $R(t)$ is the band ratio at time $t$ (DOY); $a$ is the amplitude of the sine curve; $b$ is the origin of the phase at time 0 . In addition, in Fig. 2(b), $c$ is found by taking the nearest DOY that corresponds to the maximum value of the fitted curve. To normalize seasonal band ratio values, mean summer ratio $d(\mathrm{Red} / \mathrm{SWIR})$ and mean winter ratio $e(\mathrm{Red} / \mathrm{SWIR})$ are calculated by taking the mean of all stacked summer and winter images separately using cell statistics [Fig. 2(b)]. We used the GDAL Python package to fit the sine curves on our $\mathrm{x}, \mathrm{y}, \mathrm{t}$ matrices of satellite data. 

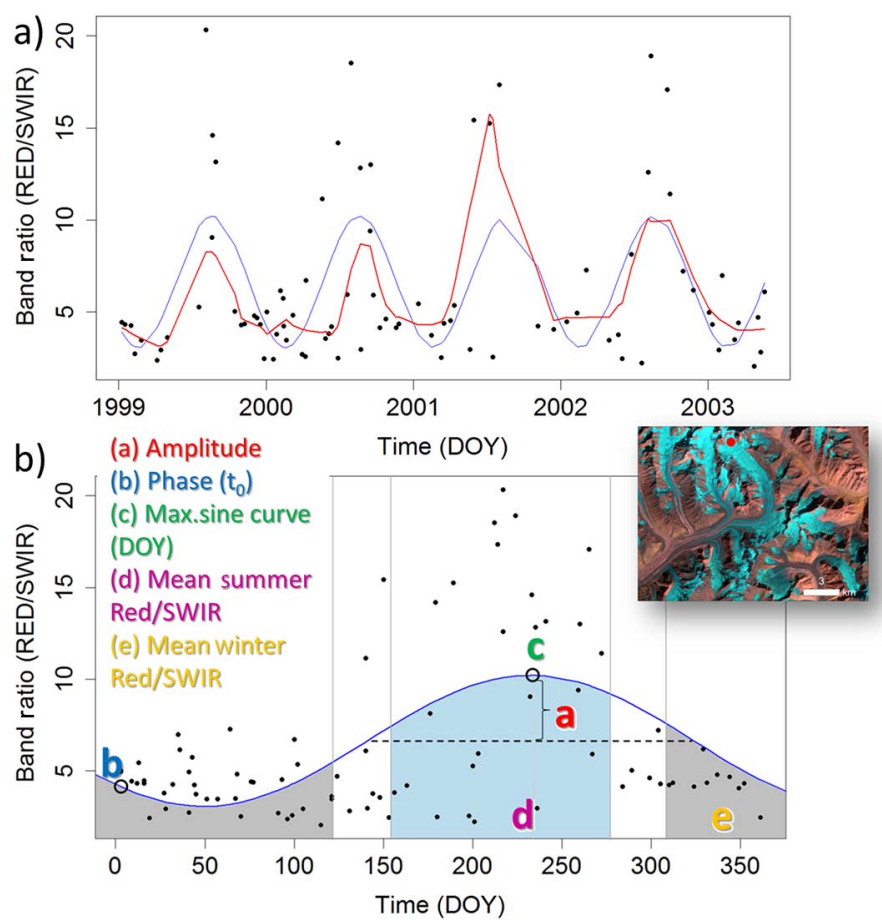

Fig. 2. (a) Sine curve (blue) fitted to band ratio values from 1999 to 2003 for one pixel in the accumulation area on a glacier in the Pamirs. An additional smoothed spline curve (red) is included to illustrate the seasonal variation in ratio values. (b) Sine curve fitted to band ratio values over several adjacent years simulating one year (104 images) for the same pixel as in (a). Parameter $a=$ amplitude, the point $c$ is the day of year corresponding to the maximum peak of the sine curve, corresponding to the phase $b$ (at time 0 ). And $d$ and $e$ are the mean ratio images for summer and winter, respectively. The Chilean dataset mean summer, $d$, is DOY 335-90 (Oct-Mar) and mean winter, $e$, is DOY 152-304 (Jun-Oct). For the Pamirs, the mean summer, $d$, is DOY: 152 273 (Jun-Sep) and mean winter, $e$, is DOY: 305-120 (Nov-Apr). Note: For this specific plot, we used point values of $3 \times 3$ pixel averaged ratio values.

\section{Application ScEnarios}

In this section, we present and discuss four application scenarios related to glacier mapping following the above introduction to the methods.

\section{A. Synthesizing an Optimal Band Ratio Image}

The multispectral band ratio method between the Red band and SWIR band (1) is typically used on single satellite scenes (e.g., [11], [12], [29]). The amount of suitable optical satellite images will increase significantly in the future, which will make it challenging and even more subjective to manually choose an optimal mapping scene from the time series. Additionally, mapping conditions may vary regionally within a single satellite scene, and this will be even more problematic for instance for a Sentinel-2 scene with $290-\mathrm{km}$ swath width, compared to Landsat's 185-km swath width (Table I). Optimal mapping conditions will thus be distributed over several scenes for different locations in a nominal acquisition frame. Here, we explore stack statistics on image composites of multitemporal optical imagery to synthesize an optimal band ratio image. Our test covers a maritime glacerized area in the Chugach Mountains in Alaska.
A band ratio image was first calculated from each time step (implicating each Landsat scene), and the mean of these stacked ratio images was calculated pixel by pixel, similar to parameter $d$ in Fig. 2. Seasonal mean mapping of snow and ice calculated from a stack of nine Landsat images is shown in Fig. 3. Regional differences in the satellite images are taken into account because each pixel in the stack of images is given new calculated values. Even though the stack mean does not neglect outliers, the temporal signature on the glacier is strong and the mean is sufficient to delineate glaciers in most circumstances. Different snow conditions might be fluctuating in mountain ranges due to climatic variations or altitude differences. The median, minimum, and maximum values showed poor results when calculated on the stack of ratio images because of this variation. In addition, these values are highly affected by cloud pixel ratio values.

The results of the synthetic ratio image is promising, especially in cloud-free areas, and also regions with various surface properties, e.g., migrating snow lines within the season (Fig. 3, insets below figure). When a cloud mask was applied on the individual band ratio images and the mean ratio image recalculated, the cloudy areas minimally affected the mapping of the glaciers [Fig. 3(b)]. The result of a synthesized ratio image is a more robust result than for many of the individual band ratio images. The advantages of the synthesized ratio images are the potential of continuity of a time series of the same glacierized area every year and potential use in glacier change analysis.

\section{B. Glacier Mapping Based on Seasonal Variations}

Here, we explore possibilities to treat each satellite image pixel as part of a time series for mapping glacier outlines. The difference from scenario [A] is that the chronological order of the image band ratios is considered for each individual pixel. Seasonal mean mapping of snow and ice is then accomplished using sinusoidal curve fitting (2). We present our results using a local and a regional study area, the Pamirs on the northern hemisphere (Fig. 4), and Chile on the southern hemisphere (Fig. 5). We illustrate the concept using the local Pamir dataset and later describe challenges when applied to a regional dataset in Chile with different climate conditions within one scene. Minimum snow and maximum snow are approximately in February/March and August for Chile, and in August/September and April for the Pamirs, respectively.

In Fig. 4, we demonstrate the Pamir study area with selected points on- and off-glacier following a centerline. This area was chosen due to the lower chance for cloud cover in this continental and high-altitude mountain environment. As explained in the method section, a sine curve (2) is fit to the Red/SWIR band ratios from the Landsat time series (Fig. 2). A time stack of 104 overlapping Landsat 5 TM and 7 ETM+ images from the Pamirs within the 1999-2003 period [Fig. 2(a), Table III] was used to simulate one year of acquisitions, in Fig. 2(b) shown by a sine curve fit to band ratio values on a glacier pixel in the accumulation area. This sine curve is calculated for each pixel in the image stack which contains a variety of surface types and cloud conditions throughout the whole year. The seasonal pattern in the ratio images was thus captured by the sine curve 


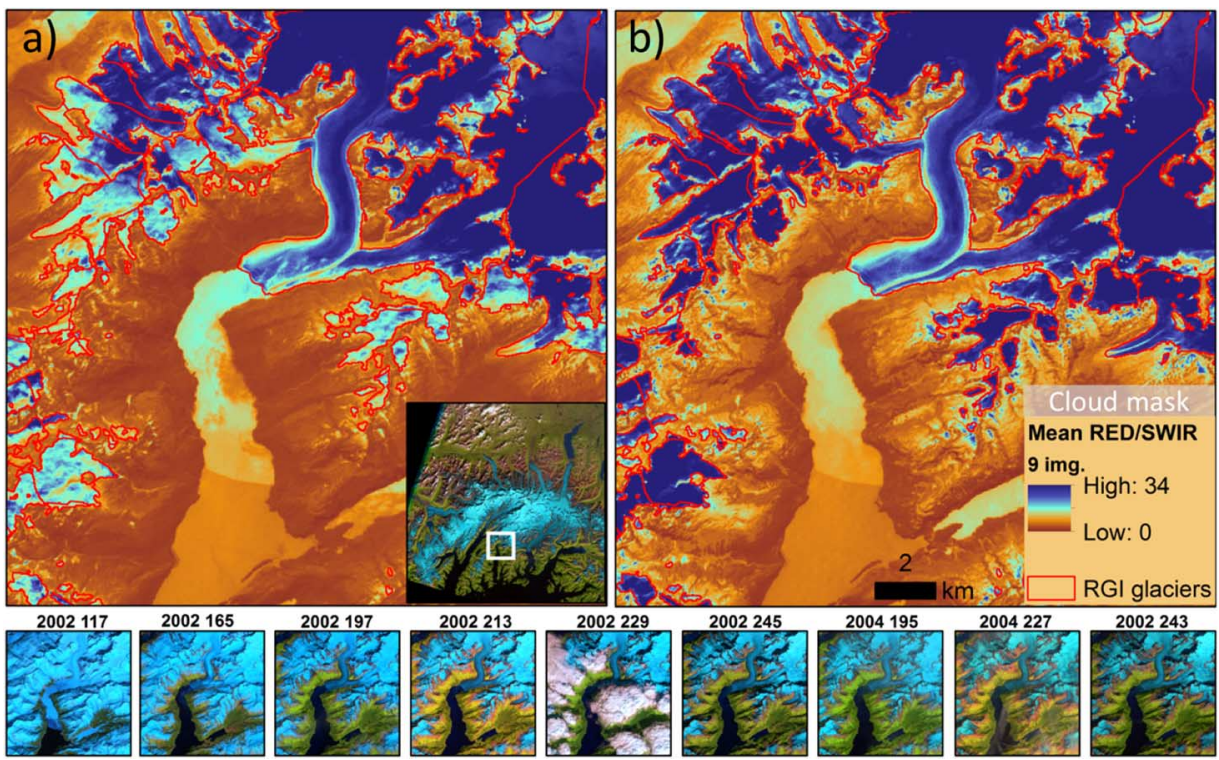

Fig. 3. Mean Red/SWIR of 9 Landsat images with various snow and cloud properties from 2002 to 2004 (Chugach Mountains in Alaska). The numbers above the 9 insets in the bottom indicate the year and DOY. (a) Without cloudmask. (b) With cloud mask [48]. Note: Debris-covered glacier ice is not taken into account, and this is why the glacier outline on the largest outlet glacier does not coincide with the RGI glacier outlines. This is qualitatively interpreted by from figure (b) where orange and brown colors fall within the RGI glacier boundary.

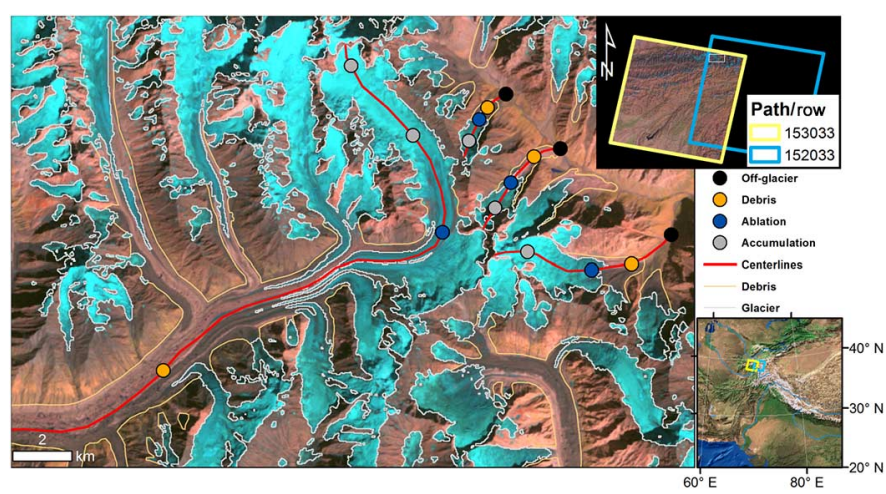

Fig. 4. Study area in the Pamirs showing the points which are used for sinecurve fitting examples in Fig. 7(c). The RGB false color composite consist of the bands SWIR, NIR, and red $(5,4,3)$. The Landsat 5 satellite image is from the year 2000 and DOY 259.

which exemplifies the potential for glacier mapping purposes [Fig. 2(b)]. The sine curve parameters exhibit different signals on- and off-glacier. The parameter $c$ is the day of year corresponding to the maximum peak of the sine curve, a measure for the phase of the sine curve alternative to the phase, $b\left(t_{0}\right)$. The histogram of parameter $c$ for the whole study area shows a clear bimodal distribution with peaks on- and off-glacier [Fig. 6(a)]. Similar results are found when the mean Red/SWIR band ratio values of the summer images are calculated, $d$ [Fig. 6(b)], a calculation similar to above application scenario [A]. Complete automatic seasonal mean mapping of snow and ice is presented in Fig. 6(c) using the mean summer ratio values. Note that all pixels through a stack of summer images selected from the 104 images are included, reflecting all surface and cloud properties. The black glacier outlines show the debris-free glacier cover based on the traditional Red/SWIR method from one optimal mapping scene. The glaciers mapped from the two methods exhibit clear similarities outlining the potential for a complete automatic mapping strategy without having to select an optimal scene. As expected, there is no clear separation between on- and off-glacier terrain in the winter ratio images [Fig. 6(b), parameter $e$ in Fig. 2(b)].

The study area in northern Patagonia in Chile has glaciers classified as temperate and wet glaciers [54], and the area experiences large gradients in precipitation and cloud cover. The strong and prevalent westerly winds bring moist air masses perpendicular into the study area, though the northern part of the study area located east of the north Patagonian ice field is in a mountain precipitation shadow. The southern part of the study area located south east of the northern Patagonian ice field is thus not protected against the moist air masses, which also results in a larger cloud cover. This area experiences one of the most extreme climate barriers in the world (e.g., [55]), making it a challenging area for mapping glaciers [30]. Glacierized areas investigated in Pamir and Chile can be seen as two end members concerning glaciological remote-sensing-based investigations. Pamir is continental, with high-elevation glaciers and relatively cold. The Chile site has a maritime climate, located at lower elevation, and has warmer glacier conditions. The regions are also located in the northern and southern hemispheres, respectively. The seasonal pattern in image ratio values is shifted between the two study areas [Fig. 7(b) and (c)], meaning that the sine curve has its maximum amplitude occurring in July/August for the Pamirs, as opposed to December/January for Chile. Chile is an extreme example of the challenges in optical satellite glacier mapping due to adverse cloud cover and snow conditions; also winter melt is common in this area [56]. Overall, the climate and mapping conditions in this area are not typical for glaciers worldwide. The meteorological conditions are also variable within the study area [54]. Our results indicate that the variation is large in space and time over the 

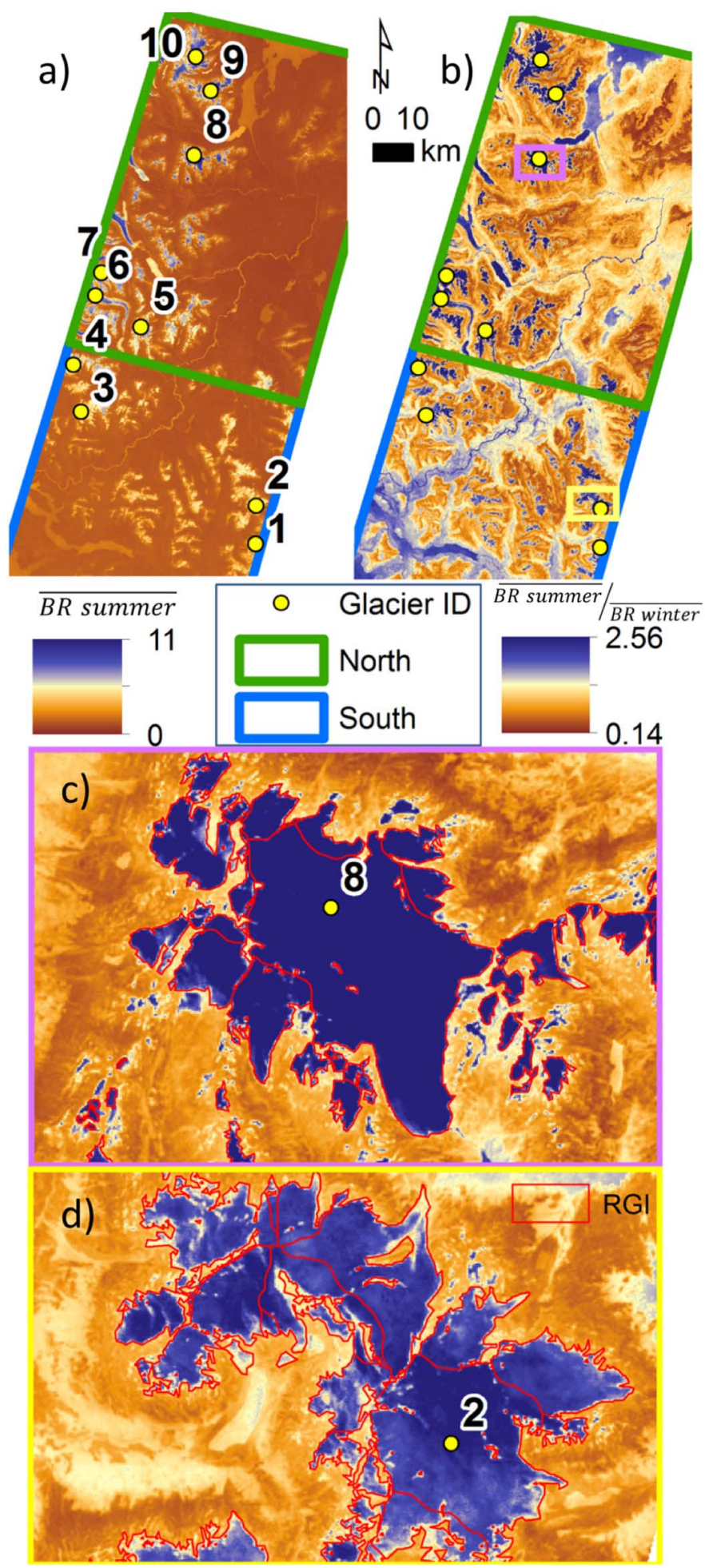

Fig. 5. (a) Mean band ratio (Red/SWIR = BR) values for summer in the Chilean study area. (b) Ratio between mean summer band ratio and mean winter band ratio. Off-glacier pixels have higher BR-values in the winter compared to summer, and on-glacier pixels have higher BR values in the summer than in winter (Off-glacier: winter $>$ summer, on-glacier: winter $<$ summer), and because of this the ratio enhances the on- and off- glacier signal. Red lines are the RGI 5.0 glacier outlines. There is a clear difference of lower and enhanced mean values on the glaciers in the (c) northern part compared to the (d) southern part of the study area, because of more frequent cloud covers in the south.
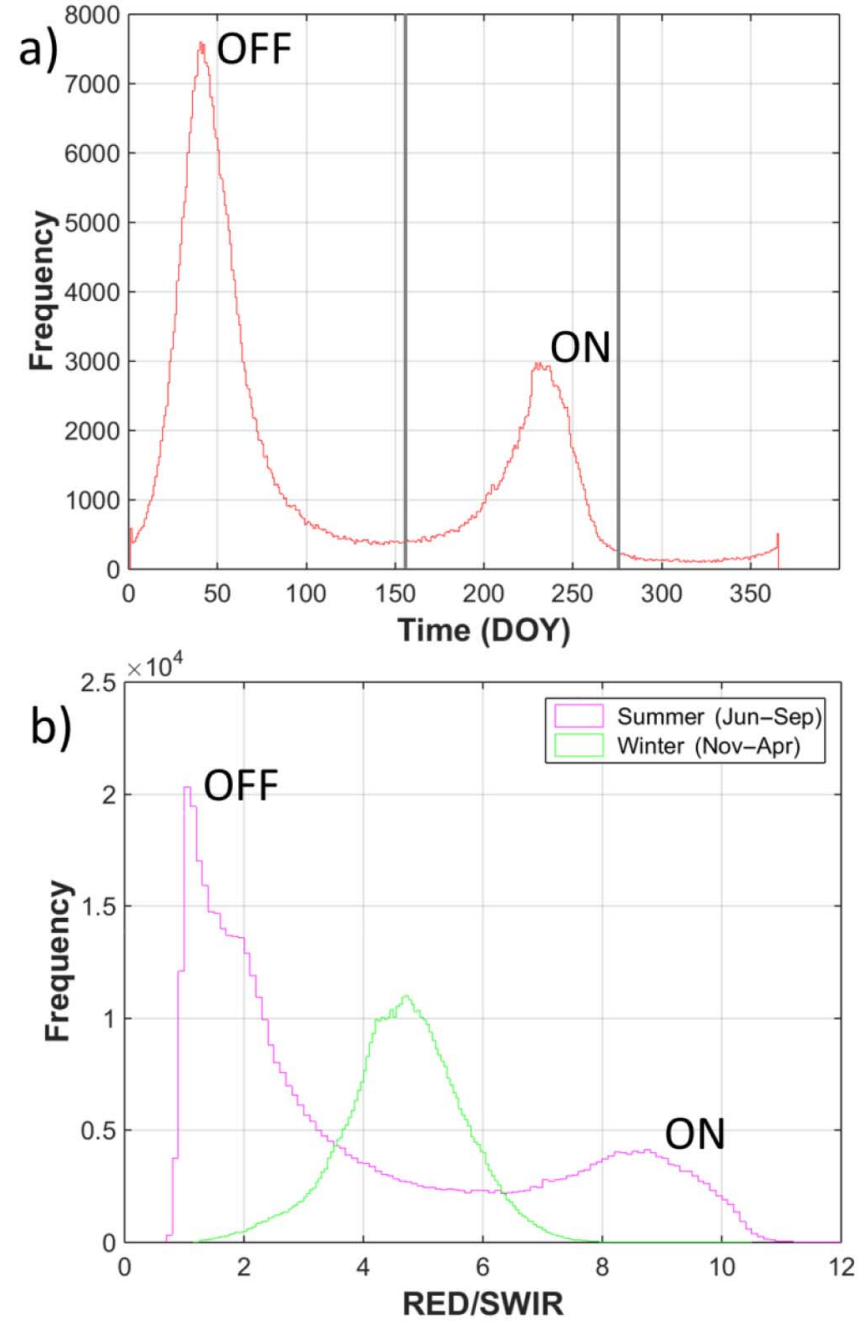

c)

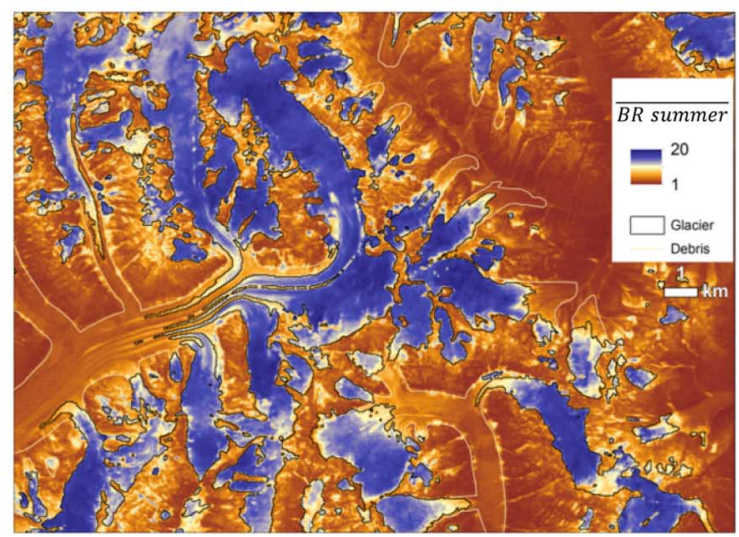

Fig. 6. Histograms form the Pamirs subset. (a) Day of year for the corresponding maximum sine curve value, based on the sine fits through the seasonal variation of the Red/SWIR band ratios. (b) Mean summer ratio values showing a similar distribution of the histogram as (a) therefore a clear difference between on- and off-glacier terrain. In (b), the mean winter ratio shows no bimodal distribution. (c) Automatic glacier (snow and ice) mapping using the mean band ratio summer values of the entire Pamir time stack [parameter $d$ in Fig. 2(b)]. Black lines indicate glacier outlines (Glacier) derived from a Landsat 5 TM image using the current glacier mapping method (Path: 153, Row:033, Year: 2000, DOY: 259) using $3 \times 3$ median filter and removed snowpatches $<8100 \mathrm{~m}^{2}$ $(3 \times 3$ pixels). Debris area (Debris) is manually digitized based on orthophotos from Google Earth from 2007 and Landsat images from 2000. 

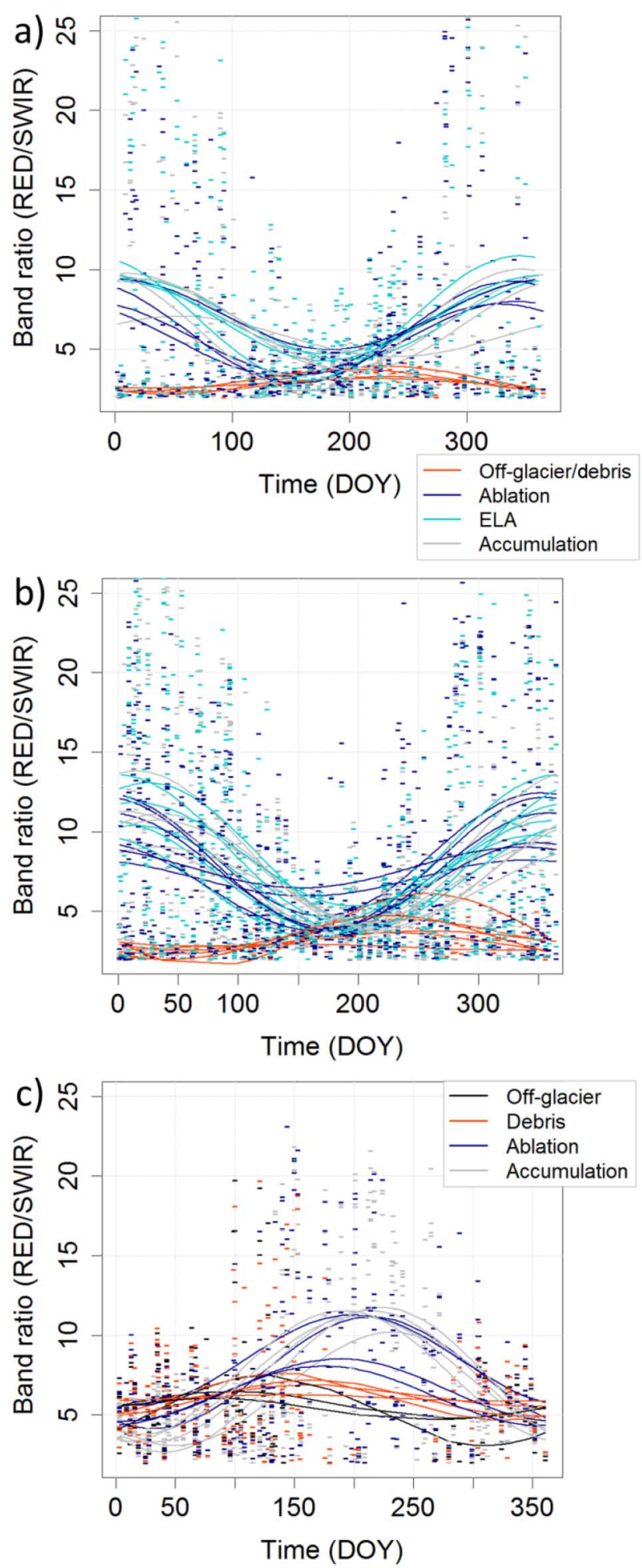

Fig. 7. Sine fits to different on- and off-glacier pixels in (a) southern Chilean subset; (b) northern Chilean subset; and (c) Pamir subset (for Pamir, the colors in the legend represent the dots presented in Fig. 4). All points represent mean Red/SWIR values of $3 \times 3$ pixels, and values of Red/SWIR $<2$ were not included. Amplitudes are higher on-glacier than outside or on debris cover. In (a) and (b), band ratio values of ELA-pixels were included. Points were found around the snow line reflected on two satellite images with good surface mapping conditions (L7: 2000068 and L7: 2002 018). The ELA points are within the elevation range of approximately $900-1500 \mathrm{~m}$ a.s.l. Note: Impurities in the ablation zone might make the sine curves less clear, e.g., two of the lower amplitude ablation sine curves in (c). study area because of local variations in climate. The Chilean study area covers a larger dataset compared to the Pamirs. As in Fig. 6(c) and application scenario [A], the mean summer ratio values (parameter $d$ in Fig. 2) were calculated for each pixel from the stacked images for the Chilean dataset [Fig. 5(a)]. The signals of the glaciers are lower in the south compared to the north due to more frequent cloud cover. In addition to the mean summer ratio values, Fig. 5(b) shows the mean of the ratio images for the summer divided by the mean ratio images for the winter. This is a way to enhance the on-and off-glacier pixel differences. (Note that this ratio is strongly related to application scenario [A].) Fig. 7(a) and (b) illustrates four glacier entities in the south of the study area, and six glacier entities in the north of the study area, respectively. A seasonal variation of the Red/SWIR values is apparent on-glacier, but not off-glacier, and the on-glacier pixels have consistently higher amplitude (ablation zone, equilibrium line altitude (ELA), and accumulation zone). Additionally, the amplitude is lower in the south [Fig. 7(a)] due to more frequent cloud cover, and this highlights that cloud cover is the largest challenge in terms of retrieval of temporal signals on glaciers in maritime climates.

The Red band is often saturated in Landsat $5 \mathrm{TM}$ and $7 \mathrm{ETM}+$, especially in the summer time, due to the 8-bit radiometric resolution. The observed seasonal signal stems from the SWIR band, and spectrally, the largest differences are found between the two bands Red/SWIR, and not NIR/SWIR, resulting in more prominent and sensitive band ratio values onglacier. The yearly variation could be related to among others temporal differences in snow properties (grain size and liquid water content), snow depth, impurities, surface roughness, or illumination angle (e.g., [57]). Most likely the most prominent reason is due to temporal changes in snow grain size during a year (e.g., [27], [29], [57], [58]). Old metamorphosed snow in the summer has lower reflectance in the SWIR band compared to fine-grained snow. The Red band is almost saturated in all snow grain sizes, but the SWIR bands show large variation between fine snow and coarse granular snow. Therefore, the Red/SWIR performs better than NIR/SWIR (even when Red is saturated), because the ratio exploits the maximum spectral differences between the two bands in the ratio, also when mapping surface types. This means the band ratio values are higher with coarse granular snow as is the case during summer. This is a pattern observed for both Pamir and Chile (Fig. 7). In sum, our analyses within application scenario [B] suggest that varying SWIR reflectance over a season from, most likely, development of grain sizes, can be a valuable parameter for exploiting satellite image time series for glacier mapping.

\section{Glacier Surface Types}

A glacier contains different surface zones or glacier surface types (e.g., [59]), and these can be classified using multispectral remote sensing (e.g., [26]). The most important boundary on a glacier is the ELA, because it divides the glacier into its main zones, the ablation and accumulation areas [60]. The ELA is not constant over a glacier as it varies a lot due to snow accumulation [59]. The transient snow line (TSL) can be used to reflect where the net surface mass balance is at zero at a 


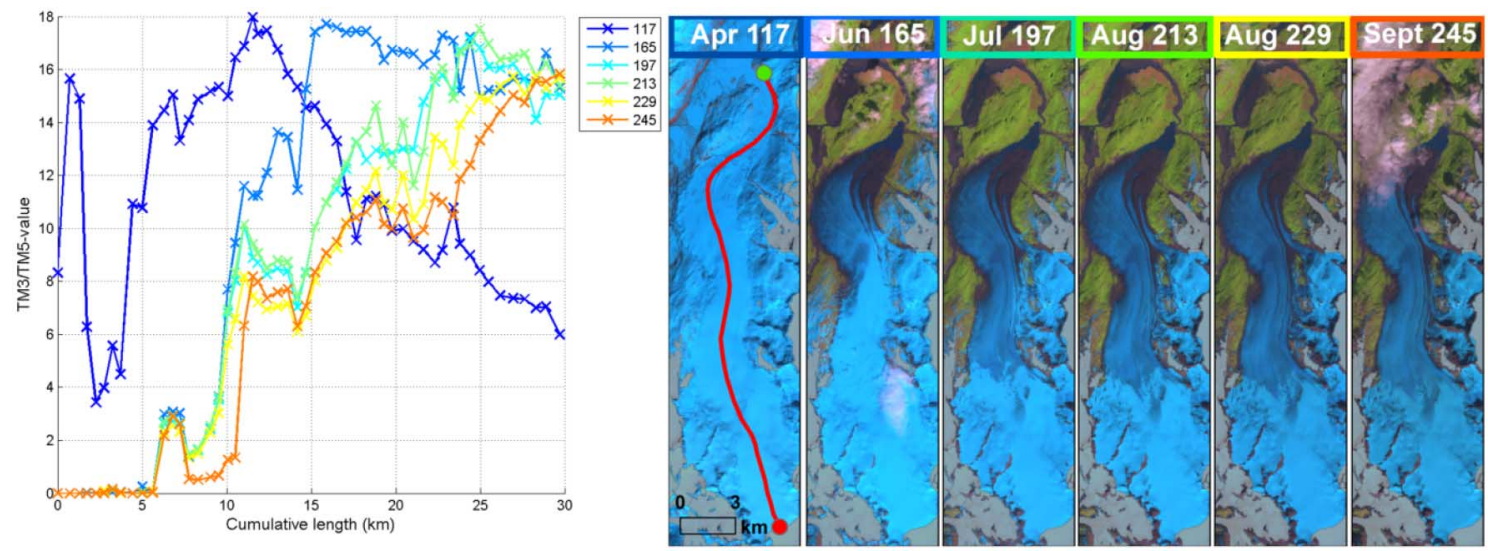

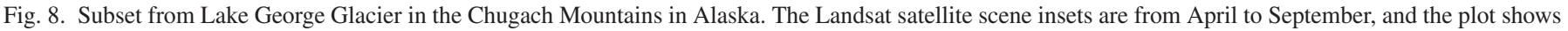

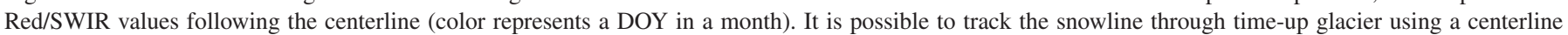

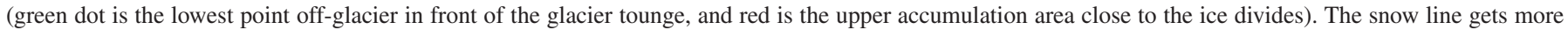
stable throughout the season and the glacier signature shows the same pattern from July to September.

particular time, and it may serve as input or validation in mass balance modeling applications (e.g., [61], [62]). The TSL can be observed using remote sensing, and especially when observing it several times within a season, one can determine where the ELA is and if it coincides with the TSL (e.g., [63], [64]). The TSL can also be used in climate studies to study spatial trends in maritime-continental transects, or topographical differences with changes in the elevation (e.g., [4], [65]). Here, we use points along a centerline and in the glacier zones, to explore the surface glacier properties or facies, with or without elevation. Two examples from Alaska and Chile are presented.

Throughout a season, glaciers display varying surface properties in the ablation and accumulation area (Fig. 8). The Red/SWIR ratio values in the April image decrease toward higher elevation (cumulative length) and into the accumulation area. Most likely the reason is different snow grain sizes in the accumulation area at this time (fine grained snow), similar to the pattern seen in the sinusoidal curve fit through the image ratio values over a year (application scenario [B]). All other months have higher ratio values in the accumulation area due to coarser snow grain size. Similar results were found by Bronge and Bronge [66] in their Antarctic study area. The snow melts away during the summer, and Fig. 8 shows that the blue ice/glacier ice has lower band ratio values than coarse snow grains (approximately ratio values estimated for our examples: $2-11$ and $11-17$, respectively). This time series shows clear spectral differences in terms of band ratio values propagating to lower values throughout the season. Even though we see this variation in ratio values, we do not find a clear distinction between the snow line and firn, in our data, since this is a transition zone where the band ratio does not give a clear separation. In very negative mass balance years, the TSL (representing ELA) is higher than the firn line, and thus, this is an important aspect that needs more investigation, preferably including ground truth. Additionally, adding information from the NIR band, which is sensitive to snow grain sizes, will potentially improve the snow and firn distinction.

To further investigate the main surface zones, we have manually picked out points from different glacier zones (ablation zone, ELA, and accumulation zone) in addition to off-glacier values. Then, we calculated the mean summer ratio values divided on the mean ratio winter values for our area in Chile [Figs. 5(b) and 9].

Fig. 9 illustrates the sensitivity of the mean band ratio summer/winter values in the study area of Chile which has different climate conditions in the south and north. The southern area has much more cloud cover compared to the northern area (as explained in application scenario [B]). The mean band ratio summer divided on mean band ratio winter values, shows a trend of higher values toward higher elevation, and a clear separation between the ablation and accumulation areas. This is an indication that the approach may be used for characterizing snow and glacier properties over large areas, for instance for automatic retrieval of TSL in the future when more satellite images become available and transects between the ablation and accumulation area can be found in the data. As in scenario $[\mathrm{B}]$, scenario $[\mathrm{C}]$ also exploited that SWIR reflectance experience a clear seasonal variation, most likely due to snow metamorphosis and related variations in grain sizes.

\section{Glacier Change Analysis}

Time series allow for investigating changing extents of glaciers over time. Bitemporal glacier change detection has long been the most common way to track glacier area change (e.g., [7]). Multitemporal change detection approaches that capture both sudden and gradual land-cover changes have been applied for vegetation and land-cover studies [52], [67]. In the future, it should be possible to exploit the seasonal band ratio pattern over glaciers described above for consecutive years to detect changes, but for this purpose, higher temporal resolution than currently available is needed.

Here, we present a multitemporal approach combining information from several years. Because of the open Landsat archive, it is possible to retrieve cloud-free Landsat images with good mapping conditions throughout the whole archive period (Table II). We neglect Landsat MSS due to the lack of a SWIR band. A false color composite of the Red/SWIR ratio images for three years $(1984,2000$, and 2011) clearly shows the retreat of the selected outlet glaciers in northern Patagonia, Chile, with 

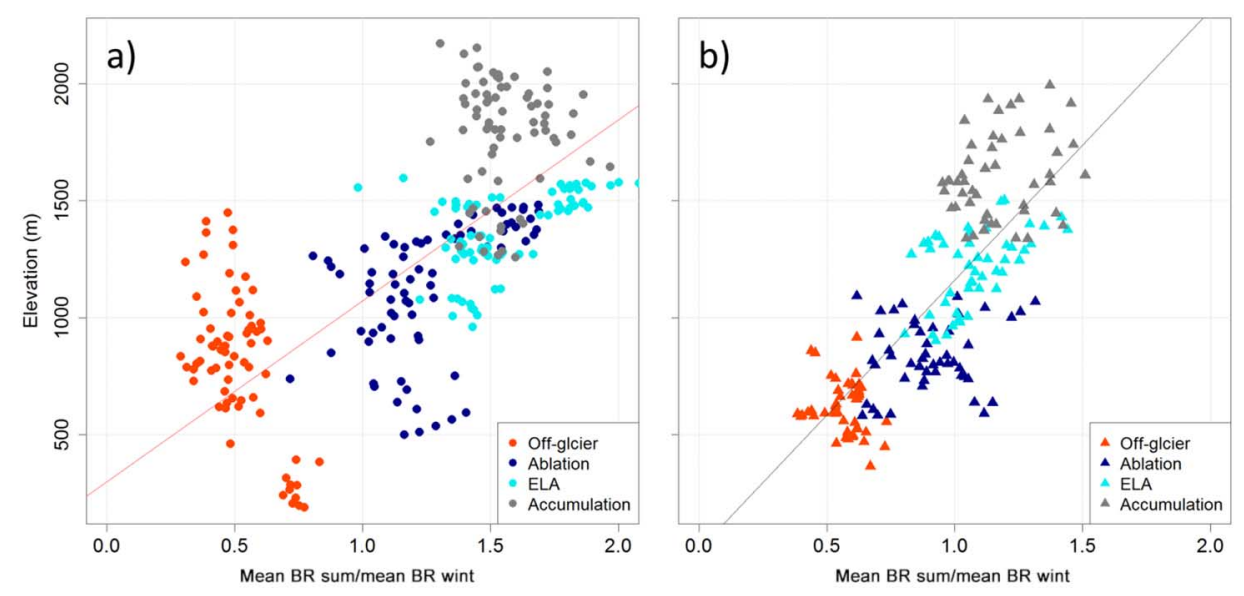

Fig. 9. Elevation is plotted with the mean summer band ratio values divided by mean winter band ratio values for (a) points off and on-glacier for the six northern glaciers in Chilean dataset and (b) same for the four southern glaciers. The glaciers in the north of the study area have a wider spectrum of the glacier signal in the band ratio values (approximately values of mean BR summer/mean BR winter for (a) are between 1 and 2, while (b) shows on-glacier values between 0.7 and 1.5). The lines show the linear regression between elevation and ratio values for (a) glacier IDs $5-10$ ( $R^{2}: 0.2744$, p-value: $\left.<2.2 \mathrm{e}-16\right)$ and (b) glacier IDs $1-4\left(\mathrm{R}^{2}: 0.3898\right.$, p-value: $\left.<2.2 \mathrm{e}-16\right)$. Note: The off-glacier orange dots and triangles are not included in the linear regression. The glacier IDs are presented in Fig. 5(a).

white representing the area where the glaciers (and snow) exist in each of the three years and elsewhere the colors reflect different surface properties in terms of glacier retreat, snow, or clouds [Fig. 10(a)]. The close up on an outlet glacier shows that this corresponds to a retreat of ca. $1 \mathrm{~km}$. The glaciers in northern Patagonia, $5^{\circ}$ latitude north of our study area, confirm this strong retreat from 1985 to 2011 [30]. The minimum and maximum values of the time series were calculated using stack statistics of five carefully selected years (1984, 2000, 2002, 2005, and 2011, Table II). Fig. 10(b) shows the ratio between maximum and minimum values as the red area, corresponding to the maximum extent of the glacier through the image stack. The minimum extent of the glaciers is represented in the turquoise colors (combined green and blue). While the glacier changes are only visualized in Fig. 10, it is straight forward to quantify the respective areas using established classification schemes. As one advantage of this maximum/minimum stack ratio method, lakes that typically disturb glacier mapping based on single scenes are not highlighted (in red) as they have not changed as rapidly as the glacier fronts [Fig. 10(b)].

In this application scenario, we have carefully searched for Landsat satellite scenes with optimal mapping conditions for each of the mapping years, due to the lack of a dense temporal stack of optical data over the test site. However, in the future, with increased temporal resolution, it could be possible to perform fully automated change analysis using for instance synthesized optimal Red/SWIR band ratio images as explained in application scenario [A]. A narrow threshold on the number of snow- and ice-free pixels in a temporal stack of band ratios could account for uncertainty in the band ratio threshold [34]. Furthermore, other potential applications of this method can be imagined, e.g., lake drainage detection due to glacier outburst floods or glacier surges.

\section{DISCUSSION}

The stacking of satellite image pixels for glacier mapping purposes has not yet been fully exploited. The application scenarios presented in this paper are nonexhaustive but reflect numerous temporal and spectral signals and thus ways of measuring glaciers through dense time series of medium-tohigh-resolution satellite images.

We have chosen not to include quantitative error estimates of the glacier mapping applications due to the challenges to actually have reliable reference data available for comparison. For example, the RGI is collected in different times than our results are gathered, and additionally, we have simulated seasonal time series using Landsat images from neighboring years. This was done because outlines are seldom overlapping in time from the same acquisition period, and the uncertainty is too large if glacier outlines from RGI are compared with a 4-year composite product.

Even though we strive toward automatic methods, it is hard not to include subjective consideration of on- and off-glacier classes in the classification process, e.g., when classifying or clustering the stacked image products to divide it into a binary product using unsupervised classification. Other more advanced methods using segmenting methods can be applied on multitemporal products, which might be necessary in highly debris-covered glacier areas [19], [20].

\section{A. Landsat Satellites and Sentinel-2}

The information available in a time series depends mainly on the temporal resolution of the satellite images. A major challenge using the Landsat TM 4 and 5 and ETM +7 satellite imagery to simulate high temporal resolution is the high amount of cloud cover in the 16-day revisit time data. Temporal curve fitting as in scenarios $[\mathrm{B}]$ and $[\mathrm{C}]$ is also challenging with irregularly spaced data in the temporal domain, but we solved this by fixing the sine curve wave length to be one year. Another challenge is the saturation in the visual bands in Landsat TM 4 and 5 and ETM+ 7 in snow and cloud pixels, mostly due to the limited radiometric resolution of 8 bits for these satellite images. The 12-bit radiometric resolution of Landsat 8 and Sentinel-2 results in fewer saturated pixels on 

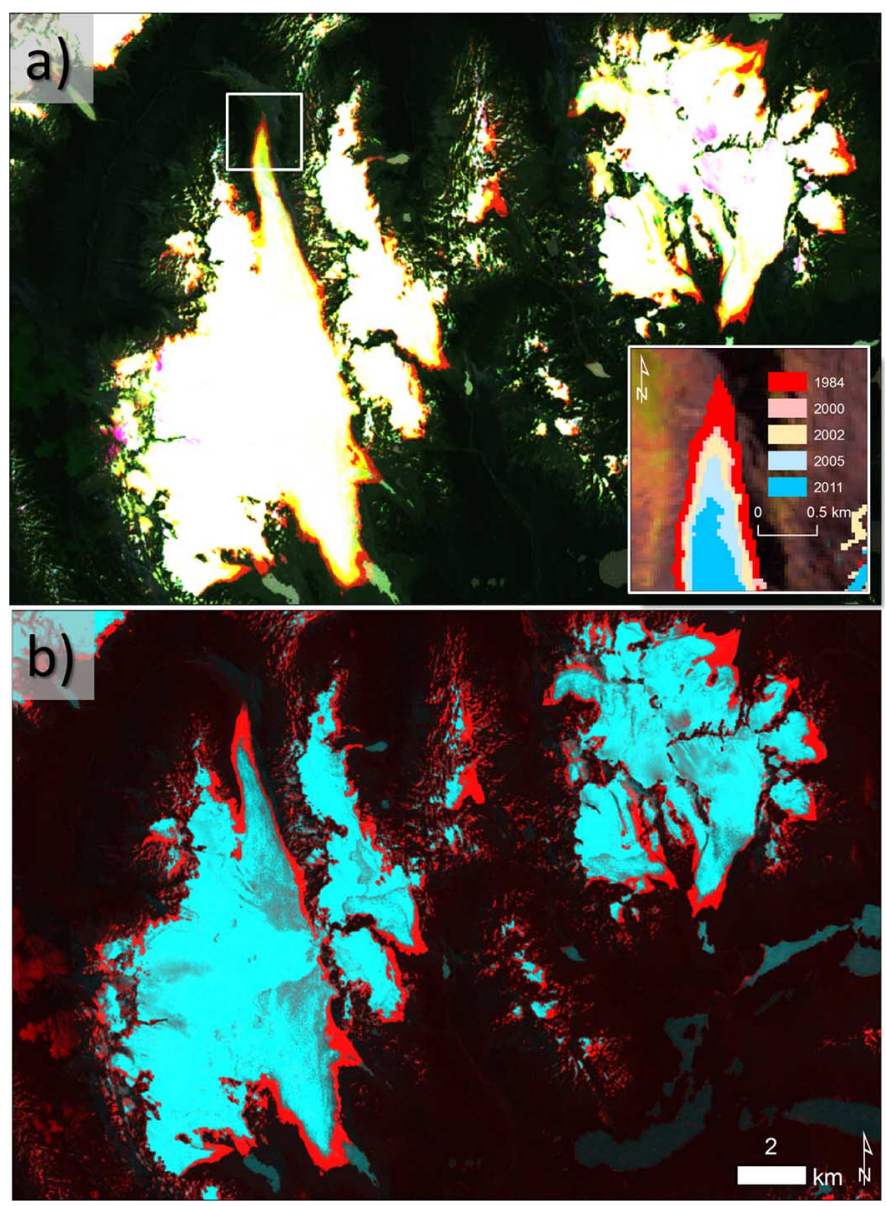

Fig. 10. (a) False color composite of ratio images from three years (R: 1984, G: 2000, and B: 2011) of mountain glaciers in Northern Patagonia, Chile. The outlet glacier has retreated almost $1 \mathrm{~km}$ in this time period (Mapped from the traditional mapping method, see inset). (b) False color composite of the ratio between maximum and minimum values (red) and minimum values (set to green and blue, showed in turquoise). Note that the lakes are not mapped when a time series is used, which is an advantage compared to the current mapping method.

the glacier in the visual Red band, thus, providing more information about the surface and snow properties in the band ratio. The improved radiometric depth also gives more information in shadowed areas [68].

\section{B. Climate, Clouds, and Ephemeral Noise}

Seasonal variation of band ratio values varies due to changing surface properties on the glacier over a year. We do not know for certain why this sinusoidal variation is prevalent (application scenario [B]), but we suggest the main reason is metamorphosis processes of snow. The solar angle, surface roughness, and steep topography are issues that make mapping of land cover like glaciers in steep mountain regions challenging even with higher temporal resolution. In land-cover studies, it is popular to use the Landsat archive for mapping or change detection (e.g., [52]), but as glacierized areas are typically located in high mountain areas, clouds are much more frequent compared to low lands. This is an important difference between the fields that must be considered when applying the same methods on different regions with different climate conditions.
With higher temporal resolution, more advanced fitting methods might be used on seasonal band ratio variations. We have applied a global fit to our data that force a sine fit with a period of one year. This simple sinusoidal model might not be able to estimate the on- and off-glacier variation in the best way possible because of large intra-annual variations (e.g., [52]), especially for snow and ice. However, when denser time series of Sentinel-2 and Landsat 8 data are available; more locally adapted fitting methods might be applied (e.g., [50], [69]).

Even though the future brings higher temporal resolution of optical satellite images, high frequency of clear observations is required. However, it should be possible to either extract cloud-contaminated pixels using cloud masks or the amount of images is so large that robust statistics can be used to limit the weighting of cloud-contaminated pixels. Ephemeral noise like atmospheric haze, mixed pixels of clouds, and surface or pixels in cloud shadow are also included in the applications of this study, but as with clouds, these effects might be reduced even more by using larger data image stacks.

\section{Processing Time and Geometric Challenges}

In the future, these application scenarios will be computationally expensive and require a lot of data storage if larger study areas and a denser time series are analyzed. For instance, calculating the mean of a subset of stacked Landsat time series takes considerable time to process. It might be helpful to mask out the glacier areas defining a buffer around the glaciers or a square, using the Randolph Glacier Inventory. Eventually, once an algorithm for automatic snow/ice mapping based on time series as in our scenario [B] becomes better established, a corresponding annual product might even be computed on the satellite data server side as a high-level product.

To stack Landsat 8 and Sentinel-2 and analyze time series on a pixel basis, is challenging in high mountain areas due to different sensor orbits and different digital terrain models used in the orthorectification process [70]. Therefore, the multisensor application must be processed carefully taking these challenges into account.

\section{CONCLUSION}

Advances and retreats of mountain glaciers in arctic and alpine areas are one of the most visible signs of change in climate, and thus, it is desirable to map glaciers more frequently. A number of improvements in glacier mapping studies will root from higher temporal resolution of medium-to-high spatial resolution satellite images, which will be increasingly available in the near future. Sentinel-2 together with Landsat 8 will improve the global data availability significantly, not least in high-latitude areas where the polar orbits converge and the density of glacierized terrain increases. The current methods of glacier mapping should be revisited to reduce dependency on single, manually chosen satellite scenes. By stacking and investigating larger time series for individual pixels, we are able to reduce noise and the chance for bias, compared to using single observations. In this study, we exemplify ways of using dense temporal stacks of satellite images for glacier 
mapping purposes. We have presented four promising application scenarios that highlight the advantages of high temporal resolution satellite imagery. By exploiting a time-series stack of images, regional differences of the temporal signature on glaciers can be reduced. One of the main signals exploited was the spectral-temporal variation of SWIR reflectance over snow and ice, most likely due to snow metamorphosis. Our results are meant as a first step, and more thorough quantitative analyses based on higher resolution time series are needed to provoke improvements and to enable the robustness of such glacier mapping to be assessed. The ultimate goal is a robust method to automatically extract repeat (clean ice) glacier areas globally by exploiting complete archives of medium-to-high-resolution multispectral satellite images (i.e., Landsat and Sentinel-2 type data).

Many future applications are possible when including and exploring the temporal dimension of new datasets. For example, there is a potential to exploit the higher temporal resolution of Sentinel-2 (combined with Landsat 8) for surveillance of glacier lake outburst floods. Further method development can be toward combining and merging the temporal signatures from optical data and other data like SAR imagery. In this study, we have exclusively focused on the spectral domain, not on exploiting feature geometry and shift as used for tracking glacier velocity [71]. The same image stacks as used here for exploiting spectral changes over time can be used to track feature offsets spatially, so that a set of glacier change variables such as extent, snow or equilibrium line, snow and ice surface properties, and ice flow could be retrieved within one system.

\section{ACKNOWLEDGMENT}

Landsat TM/ETM+ scenes were downloaded from Earth Explorer (http://earthexplorer.usgs.gov/). The RGI glacier outlines were obtained from the Global Land Ice Measurements from Space (GLIMS: http://www.glims.org/RGI/). The SRTM DEM was provided by EROS data center at the US Geological Survey. The authors would like to thank B. Altena (UiO) for his comments on the work, and discussions about the datasets and the spectral properties of snow. They would also like to thank all colleagues at the GEOHYD-section (UiO) for feedbacks and fruitful discussions. This is publication No.75 of the Nordic Centre of Excellence SVALI, "Stability and Variations of Arctic Land Ice," funded by the Nordic Top-level Research Initiative (TRI). All authors developed the concepts of the study. S. $\mathrm{H}$. Winsvold prepared and analyzed the data. She wrote the manuscript with contribution from A. Kääb and C. Nuth.

\section{REFERENCES}

[1] D. Vaughan et al., "Observations: Cryosphere," in Climate Change 2013: The Physical Science Basis. Contribution of Working Group I to the Fifth Assessment Report of the Intergovernmental Panel on Climate Change, vol. 1, T. F. Stocker et al. Ed. Cambridge, U.K.: Cambridge Univ. Press, 2013, pp. 1-2.

[2] R. G. Barry, "The status of research on glaciers and global glacier recession: A review," Progr. Phys. Geogr., vol. 30, pp. 285-306, 2006.
[3] R. Hock, "Glaciers and climate change," in Global Environmental Change. New York, NY, USA: Springer, 2014, pp. 205-210.

[4] A. Rabatel, A. Letréguilly, J. Dedieu, and N. Eckert, "Changes in glacier equilibrium-line altitude in the western Alps from 1984 to 2010: Evaluation by remote sensing and modeling of the morphotopographic and climate controls," Cryosphere, vol. 7, pp. 1455-1471, 2013.

[5] J. McCarthy, O. Canziani, N. Leary, D. Dokken, and K. White, Climate Change 2001: Impacts, Adaptations, and Vulnerability. Cambridge, U.K.: Cambridge Univ. Press, 2001.

[6] B. Marzeion, A. Jarosch, and M. Hofer, "Past and future sea-level change from the surface mass balance of glaciers," Cryosphere, vol. 6, pp. 12951322, 2012.

[7] S. H. Winsvold, L. M. Andreassen, and C. Kienholz, "Glacier area and length changes in norway from repeat inventories," Cryosphere, vol. 8, pp. 1885-1903, 2014.

[8] L. Andreassen, M. Huss, K. Melvold, H. Elvehøy, and S. Winsvold, "Ice thickness measurements and volume estimates for glaciers in norway," $J$. Glaciol., vol. 61, pp. 763-775, 2015.

[9] W. Haeberli, "Glaciers and Ice Caps: Historical Background and Strategies of World-Wide Monitoring. Ed. Cambridge, U.K.: Cambridge Univ. Press, 2004, pp. 559-578.

[10] F. Paul, A. Kääb, and W. Haeberli, "Recent glacier changes in the Alps observed by satellite: Consequences for future monitoring strategies," Global Planet. Change, vol. 56, pp. 111-122, 2007.

[11] F. Paul et al., "Recommendations for the compilation of glacier inventory data from digital sources," Ann. Glaciol., vol. 50, pp. 119-126, 2010.

[12] A. E. Racoviteanu, F. Paul, B. Raup, S. J. S. Khalsa, and R. Armstrong, "Challenges and recommendations in mapping of glacier parameters from space: Results of the 2008 global land ice measurements from space (GLIMS) workshop, boulder, colorado, USA," Ann. Glaciol., vol. 50, pp. 53-69, 2010.

[13] K. J. Bayr, D. Hall, and W. Kovalick, "Observations on glaciers in the eastern austrian alps using satellite data," Int. J. Remote Sens., vol. 15, pp. 1733-1742, 1994.

[14] L. Andreassen, S. H. Winsvold, F. Paul, and J. Hausberg, "Inventory of norwegian glaciers," NVE, Oslo, Norway, NVE 38, 2012, p. 236.

[15] F. Paul, A. Kääb, M. Maisch, T. Kellenberger, and W. Haeberli, "The new remote-sensing-derived swiss glacier inventory: I. methods," Ann. Glaciol., vol. 34, pp. 355-361, 2002.

[16] D. K. Hall, G. A. Riggs, and V. V. Salomonson, "Development of methods for mapping global snow cover using moderate resolution imaging spectroradiometer data," Remote Sens. Environ., vol. 54, pp. 127-140, 1995.

[17] A. E. Racoviteanu, Y. Arnaud, M. W. Williams, and J. Ordonez, "Decadal changes in glacier parameters in the cordillera blanca, peru, derived from remote sensing," J. Glaciol., vol. 54, pp. 499-510, 2008.

[18] P. Burns and A. Nolin, "Using atmospherically-corrected Landsat imagery to measure glacier area change in the cordillera blanca, peru from 1987 to 2010," Remote Sens. Environ., vol. 140, pp. 165-178, 2014.

[19] P. Rastner, T. Bolch, C. Notarnicola, and F. Paul, "A comparison of pixeland object-based glacier classification with optical satellite images," IEEE J. Sel. Topics Appl. Earth Observ. Remote Sens., vol. 7, no. 3, pp. 853-862, Mar. 2014.

[20] B. A. Robson, C. Nuth, S. O. Dahl, D. Hölbling, T. Strozzi, and P. R. Nielsen, "Automated classification of debris-covered glaciers combining optical, SAR and topographic data in an object-based environment," Remote Sens. Environ., vol. 170, pp. 372-387, 2015.

[21] J. L. Engle and O. Weinstein, "The thematic mapper-an overview," IEEE Trans. Geosci. Remote Sens., vol. 21, no. 3, pp. 258-265, Jul. 1983.

[22] S. N. Goward, J. G. Masek, D. L. Williams, J. R. Irons, and R. Thompson, "The Landsat 7 mission: Terrestrial research and applications for the 21st century," Remote Sens. Environ., vol. 78, pp. 3-12, 2001.

[23] M. Drusch et al., "Sentinel-2: ESA's optical high-resolution mission for GMES operational services," Remote Sens. Environ., vol. 120, pp. 25-36, 2012.

[24] D. P. Roy et al., "Landsat-8: Science and product vision for terrestrial global change research," Remote Sens. Environ., vol. 145, pp. 154-172, 2014.

[25] Z. Zhu, S. Wang, and C. E. Woodcock, "Improvement and expansion of the Fmask algorithm: Cloud, cloud shadow, and snow detection for Landsats 4-7, 8, and sentinel 2 images," Remote Sens. Environ., vol. 159, pp. 269-277, 2015.

[26] A. Pope and W. G. Rees, "Impact of spatial, spectral, and radiometric properties of multispectral imagers on glacier surface classification," Remote Sens. Environ., vol. 141, pp. 1-13, 2014. 
[27] S. G. Warren, "Optical properties of snow," Rev. Geophysics and Space Phys., vol. 20, no. 1, pp. 67-89, 1982.

[28] J. Dozier and D. Marks, "Snow mapping and classification from Landsat thematic mapper data," Ann. Glaciol., vol. 9, pp. 97-103, 1987.

[29] J. Dozier, "Spectral signature of alpine snow cover from the landsat thematic mapper," Remote Sens. Environ., vol. 28, pp. 9-22, Apr./Jun. 1989.

[30] F. Paul and N. Mölg, "Hasty retreat of glaciers in northern Patagonia from 1985 to 2011," J. Glaciol., vol. 60, pp. 1033-1043, 2014.

[31] Q. Ye, S. Kang, F. Chen, and J. Wang, "Monitoring glacier variations on geladandong mountain, central tibetan plateau, from 1969 to 2002 using remote-sensing and GIS technologies," J. Glaciol., vol. 52, pp. 537-545, 2006.

[32] Q. Ye, T. Yao, S. Kang, F. Chen, and J. Wang, "Glacier variations in the naimona'nyi region, western himalaya, in the last three decades," Ann. Glaciol., vol. 43, pp. 385-389, 2006.

[33] F. Paul, "Revealing glacier flow and surge dynamics from animated satellite image sequences: Examples from the karakoram," Cryosphere Discuss., vol. 9, pp. 2597-2623, 2015.

[34] D. J. Selkowitz and R. R. Forster, "An automated approach for mapping persistent ice and snow cover over high latitude regions," Remote Sens., vol. 8, p. 16, 2016.

[35] R. W. Sidjak and R. D. Wheate, "Glacier mapping of the Illecillewaet icefield, british columbia, canada, using Landsat TM and digital elevation data," Int. J. Remote Sens., vol. 20, pp. 273-284, 1999.

[36] A. Kääb, Remote Sensing of Mountain Glaciers and Permafrost Creep, Physische Geographie, Dept. Geography, Zurich, vol. 48, 2005, p. 266.

[37] N. Otsu, "A threshold selection method from gray-level histograms," Automatica, vol. 11, pp. 23-27, 1975.

[38] F. Paul, C. Huggel, and A. Kääb, "Combining satellite multispectral image data and a digital elevation model for mapping debris-covered glaciers," Remote Sens. Environ., vol. 89, pp. 510-518, 2004.

[39] H. Frey, F. Paul, and T. Strozzi, "Compilation of a glacier inventory for the western himalayas from satellite data: Methods, challenges, and results," Remote Sens. Environ., vol. 124, pp. 832-843, 2012.

[40] T. Smith, B. Bookhagen, and F. Cannon, "Improving semi-automated glacier mapping with a multi-method approach: Applications in central asia," Cryosphere, vol. 9, pp. 1747-1759, 2015.

[41] USGS. (2015, Oct. 18). Landsat Science Team Meeting [Online]. Available:http://landsat.usgs.gov/science_LST_july2015.php

[42] B. Holben and C. Justice, "An examination of spectral band ratioing to reduce the topographic effect on remotely sensed data," Int. J. Remote Sens., vol. 2, pp. 115-133, 1981.

[43] W. T. Pfeffer et al., "The randolph glacier inventory: A globally complete inventory of glaciers," J. Glaciol., vol. 60, pp. 537-552, 2014.

[44] F. Paul et al., "The glaciers climate change initiative: Methods for creating glacier area, elevation change and velocity products," Remote Sens. Environ., vol. 162, pp. 408-426, 2013.

[45] F. Paul et al., "On the accuracy of glacier outlines derived from remotesensing data," Ann. Glaciol., vol. 54, pp. 171-182, 2013.

[46] F. Paul and A. Kääb, "Perspectives on the production of a glacier inventory from multispectral satellite data in arctic Canada: Cumberland peninsula, baffin island," Ann. Glaciol., vol. 42, pp. 59-66, 2005.

[47] F. Paul and L. M. Andreassen, "A new glacier inventory for the svartisen region, norway, from Landsat ETM + data: Challenges and change assessment," J. Glaciol., vol. 55, pp. 607-618, 2009.

[48] L. Andreassen, F. Paul, A. Kääb, and J. Hausberg, "Landsat-derived glacier inventory for Jotunheimen, norway, and deduced glacier changes since the 1930s," Cryosphere, vol. 2, pp. 131-145, 2008.

[49] Z. Zhu and C. E. Woodcock, "Object-based cloud and cloud shadow detection in Landsat imagery," Remote Sens. Environ., vol. 118, pp. 8394, 2012.

[50] P. Jönsson and L. Eklundh, "Seasonality extraction by function fitting to time-series of satellite sensor data," IEEE Trans. Geosci. Remote Sens., vol. 40, no. 8, pp. 1824-1832, Aug. 2002.

[51] Z. Zhu, C. E. Woodcock, and P. Olofsson, "Continuous monitoring of forest disturbance using all available Landsat imagery," Remote Sens. Environ., vol. 122, pp. 75-91, 2012.

[52] Z. Zhu and C. E. Woodcock, "Continuous change detection and classification of land cover using all available Landsat data," Remote Sens. Environ., vol. 144, pp. 152-171, 2014.

[53] Z. Zhu, C. E. Woodcock, C. Holden, and Z. Yang, "Generating synthetic Landsat images based on all available Landsat data: Predicting Landsat surface reflectance at any given time," Remote Sens. Environ., vol. 162, pp. $67-83,2015$
[54] E. Sagredo and T. Lowell, "Climatology of Andean glaciers: A framework to understand glacier response to climate change," Global Planet. Change, vol. 86, pp. 101-109, 2012.

[55] J. C. Aravena and B. H. Luckman, "Spatio-temporal rainfall patterns in southern south america," Int. J. Climatol., vol. 29, pp. 2106-2120, 2009.

[56] B. W. Brock, F. Burger, A. Rivera, and A. Montecinos, "A fifty year record of winter glacier melt events in southern Chile, $38^{\circ}-42^{\circ} \mathrm{S}$," Environ. Res. Lett., vol. 7, p. 9, 2012.

[57] D. Scherer et al., "Remote sensing of snow cover," Geophys. Monogr. Amer. Geophys. Union, vol. 163, p. 7, 2005.

[58] C. E. Bøggild, Personal Communication, Technical Univ. Denmark (DTU), Dept. Civil Eng., Jun. 2015.

[59] D. Benn and D. J. Evans, Glaciers and Glaciation. Evanston, IL, USA Routledge, 2014.

[60] J. Cogley et al., "Glossary of glacier mass balance and related terms," IHP-VII Tech. Doc. Hydrology No. 86, IACS Contribution No. 2, UNESCO-IHP, Paris, 2011.

[61] J. Hulth, C. Rolstad Denby, and R. Hock, "Estimating glacier snow accumulation from backward calculation of melt and snowline tracking," Ann. Glaciol., vol. 54, pp. 1-7, 2013

[62] M. Réveillet, A. Rabatel, F. Gillet-Chaulet, and A. Soruco, "Simulation of changes to glaciar zongo, bolivia (16 S), over the 21 st century using a 3-D full-Stokes model and CMIP5 climate projections," Ann. Glaciol., vol. 56, p. 89, 2015.

[63] G. Østrem, "The transient snowline and glacier mass balance in southern British columbia and alberta, canada," Geografiska Annaler. Ser. A. Phys. Geogr., vol. 55, pp. 93-106, 1973.

[64] A. Rabatel et al., "Can the snowline be used as an indicator of the equilibrium line and mass balance for glaciers in the outer tropics?," J. Glaciol., vol. 58, pp. 1027-1036, 2012.

[65] G. Østrem, "The height of the glaciation limit in southern british columbia and alberta," Ser. A Phys. Geogr., vol. 48, pp. 126-138, 1966.

[66] L. B. Bronge and C. Bronge, "Ice and snow-type classification in the vestfold hills, east antarctica, using Landsat-TM data and ground radiometer measurements," Int. J. Remote Sens., vol. 20, pp. 225-240, 1999.

[67] J. N. Hird, G. Castilla, G. J. McDermid, and I. T. Bueno, "A simple transformation for visualizing non-seasonal Landscape change from dense time series of satellite data," IEEE J. Sel. Topics Appl. Earth Observ. Remote Sens., pp. 1-12, Apr. 2015.

[68] F. Paul, S. H. Winsvold, A. Kääb, G. Bippus, and T. Nagler, "Glacier remote sensing using sentinel-2. Part II: Mapping glacier extents, surface facies, and comparison to Landsat 8," unpublished.

[69] P. M. Atkinson, C. Jeganathan, J. Dash, and C. Atzberger, "Intercomparison of four models for smoothing satellite sensor time-series data to estimate vegetation phenology," Remote Sens. Environ., vol. 123 , pp. 400-417, 2012.

[70] A. Kääb, S. H. Winsvold, B. Altena, C. Nuth, T. Nagler, and J. Wuite, "Glacier remote sensing using sentinel-2. Part I: Radiometric and geometric performance, application to ice velocity, and comparison to Landsat $8, "$ unpublished.

[71] T. Heid and A. Kääb, "Evaluation of existing image matching methods for deriving glacier surface displacements globally from optical satellite imagery," Remote Sens. Environ., vol. 118, pp. 339-355, 2012.

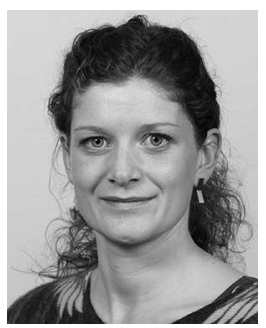

Solveig Havstad Winsvold received the M.S. degree in geography and geoinformatics from the University of Copenhagen, Copenhagen, Denmark, in 2009. She is currently working toward the Ph.D. degree at the Institute for Geosciences, University of Oslo, Oslo, Norway.

Her main focus in the Ph.D. degree is to explore the potential of new glacier mapping methods taking advantage of high-temporal resolution time series of both optical and SAR imagery. From 2009 to 2012, she was with the Norwegian Water Resources and Energy Directorate (NVE), Oslo, Norway, at the section for glacier, ice, and snow. Her research interests include mapping the Norwegian glaciers using medium-to-high resolution optical data in addition to previous analogue maps. This work resulted in three glacier inventories going back to the $1950 \mathrm{~s}$, and made her a coeditor on the book on Inventory of Norwegian Glaciers. Additionally she published a glacier area and length change analysis of all glaciers in Norway based on the above mentioned datasets. She is currently a part of the Sentinel-2 Expert team analyzing the applicability of Sentinel-2 images for glacier mapping purposes. 


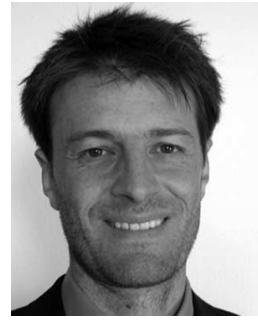

Andreas Kääb (M'07) received the Diploma degree in geomatics engineering from the Technical University of Munich, Munich, Germany, in 1990, the Ph.D. degree in earth sciences from the ETH Zurich, Zurich, Switzerland, in 1996, and the Habilitation degree in physical geography from the University of Zurich, Zurich, Switzerland, in 2004.

He is currently a Professor of Remote Sensing with the Department of Geosciences, University of Oslo, Oslo, Norway. He has authored over 100 articles and a book on Remote Sensing of Mountain Glaciers and Permafrost Creep, coedited a book on Glacier Remote Sensing, a further one on High Mountain Cryosphere Risks. His research interests include the observation of climate change impacts on glaciers, permafrost, and the hazards related to these.

Dr. Kääb is a coeditor of the journal The Cryosphere.

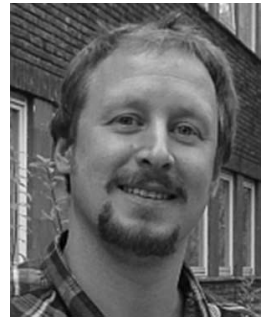

Christopher Nuth received the B.S. degree in environmental science from McGill University, Montreal, QC, Canada, in 2004, the M.S. degree (2007) and the Ph.D. degree (2011) in geosciences from the University of Oslo, Oslo, Norway, focusing on glacier remote sensing and application of high-precision geomatic techniques for observing glacier change.

He has been employed as a Researcher for the European Space Agency and involved in a number of EU and Norwegian Research Council funded projects focusing on glaciers, the cryosphere, geomatics and remote sensing, and geophysical applications for observing glaciers. He has been studying the glaciers of Svalbard through in situ field observations of the past 10 years and focuses on applying his ground-based knowledge to extract essential glaciological and climatic information from satellite data for global application. 